\title{
THE BRITISH EMPIRE CANCER CAMPAIGN
}

\author{
By DR. E. Boyland, \\ The Royal Cancer Hospital, London
}

\section{$\mathrm{T}$} HE publication of the sixteenth annual report of the British Empire Cancer Campaign was very little delayed by the outbreak of hostilities, and the report has appeared in its usual form and is actually larger than it has ever been before. During the War as much cancer research as possible will continue, so long as the scientific workers concerned are not all required for emergency work. There is no doubt that the greatest progress in cancer research has been made in Great Britain and in the United States. Government support and large endowments in the United States are likely to increase American cancer research in the future, and it is to be hoped that the British effort, will not fall off.

Among the clinical accounts in this report is a description by Mr. J. P. Lockhart-Mummery and Dr. C. E. Dukes of the familial adenomatosis of the colon and rectum and its relationship to cancer. Adenomatosis is an inherited disease, which is not generally manifested until childhood or puberty. The malignant disease which frequently develops from this adenomatosis occurs at a relatively early age and often has multiple foci of carcinoma. The adenomatosis appears to be transmitted by both sexes, probably as a Mendelian character.

Much of the clinical work describes the results of radiological treatment. Such material is given in the reports of the Westminster Hospital, St. Bartholomew's Hospital, the Marie Curie Hospital and the Mount Vernon Hospital. One interesting development of research on radiations is the discovery of the rhythmic or periodic variation in their action on colloids. If colloidal solutions of carbon, graphite, quartz, gold or albumin are irradiated with a certain dose of neutrons, X-rays, $\gamma$-rays or by ultra-violet light, they are coagulated, but if the dose is increased by a certain amount no coagulation occurs. With increasing amounts of radiation, the effect on coagulation alternately increases to a maximum and then falls to zero. This appears to be a periodic phenomenon analogous to the formation of Liesegang rings, and it would be extremely interesting to know if the same periodic variation occurs in the irradiation of living tissues.

At the recently formed Cancer Research Centre of the University of Oxford, Dr. I. Berenblum, Dr. E. Chain and Dr. N. G. Heatley have investigated the metabolic activities of very small amounts of surviving skin epithelium in a micro-respiro- meter. This tissue shows aerobic glycolysis and has a respiratory quotient of $0 \cdot 7$, properties which at one time were considered to be peculiar to tumour tissues. In fact, the current report from the Royal Victoria Infirmary, Newcastle-on-Tyne, contains the following rather misleading statement. "There appear at the present time to be two main points in which the metabolism of cancer differs from that of most normal tissues. First the ability of cancer cells to form lactic acid persists even when the tissue is respiring, secondly cancer tissue has a respiratory quotient indicating that the oxidation of carbohydrate is abnormal." Since Warburg discovered that slices of malignant tissues produced lactic acid from glucose in the presence of oxygen, retina, smooth muscle, striped muscle, liver, cartilage, bone marrow, lymph glands, kidney medulla and skin have also been shown to glycolyse aerobically. It is therefore impossible to consider this characteristic to be peculiar to tumours. Neither is the lowered respiratory quotient specifically characteristic of malignant tissues.

It was known that the magnitude of respiration and glycolysis did not vary greatly in tumours of different rates of growth, and now Dr. E. Boyland and Mrs. M. E. Boyland have shown that this holds even with enormous variations in growthrate and that it is possible to inhibit the growth of sarcomata in mice without significantly reducing the respiration and glycolysis as measured in vitro. Thus the largest metabolic processes do not vary with rate of growth; this suggests that some tumours must be extremely inefficient in obtaining energy for growth from carbohydrate.

Experiments on carcinogenesis are described in several of the reports. At St. Bartholomew's Hospital, $1: 2: 5: 6$-dibenzanthracene in olive oil was introduced into the stomachs of mice in an attempt to produce cancer of the alimentary tract. No alimentary tumours were produced but 95 per cent of those mice which survived the period of injection died with pulmonary tumours. Only minute amounts of the carcinogenic compound could have reached the lungs, and the experiment indicates that the lungs are very sensitive to the tumour-inducing action of dibenzanthracene and that the tissues of the alimentary canal are very resistant.

Other experiments showing the amounts of carcinogenic agent necessary for tumour production were carried out by Dr. P. M. H. Browning at 
Glasgow. In these the injection of $0.0025 \mathrm{gm}$. of 2 (p-amino-styryl) 6 ( $p$-acetylamino benzoylamino) quinoline methoacetate ('Styryl 430 ') into mice was necessary to produce sarcomata, while even with $0.0005 \mathrm{gm}$. a small coloured depot may be found more than a year after injection. Many other trypanocidal compounds related to Styryl 430 have been tested, but as yet this is the only known compound of its type with carcinogenic activity.

Mr. J. C. Mottram, at the Mount Vernon Hospital, has used the sensitivity of Infusoria to demonstrate the photo-oxidation of carcinogenic compounds. On the whole, the carcinogenic compounds are easily oxidized in ultra-violet light to water-soluble toxic products, and there is rough but not exact parallelism between this action and carcinogenic power. Similar results were obtained some years ago by Dr. E. Boyland, who examined the effect of photo-oxidation products on the enzyme lactic dehydrogenase. The chemical changes which carcinogens undergo in ultra-violet light in the presence of oxygen are still obscure, but Prof. J. W. Cook and Mr. R. Martin of the Royal Cancer Hospital have isolated the photooxide of $9: 10$-dimethyl-1 : 2 -benzanthracene. The parent hydrocarbon, $9: 10$-dimethyl-1 : 2-benzanthracene, has very high carcinogenic activity, producing epithelial tumours in mice in thirty-five days ; $5: 9: 10$-trimethyl-1:2-benzanthracene, however, is now the most active compound and has given skin tumours in thirty-one days in tests carried out at the Royal Cancer Hospital. Derivatives of $1: 2$-benzanthracene with substituents in the 9 and 10 (meso) positions not only give more stable photo-oxides but also more stable maleic anhydride derivatives; thus Dr. L. D. Parsons and Mr. F. L. Warren find that the $\alpha$ - $\beta$-endosuccinate of 9:10-dimethyl-1:2-benzanthracene is very stable and is not carcinogenic, while the corresponding derivative of methylcholanthrene dissociates and is actively carcinogenic. These results indicate that the action of these watersoluble carcinogenic compounds is due to their ability to dissociate and liberate the parent hydrocarbon after injection rather than to the action of the water-soluble compound per se. These watersoluble carcinogenic compounds cause lysis of red blood cells in vitro, but this action is also shown by similar derivatives of some compounds which have no carcinogenic action.

Dr. P. A. Gorer at the Lister Institute has investigated immunity within pure lines of inbred mice. While it is relatively easy to produce immunity to a tumour in mixed stock animals it is extremely difficult to demonstrate immunity within a pure line. With two types of leukæmia which have been maintained in the pure lines in which they arose, it has not been possible to produce immunity within the homologous pure strain. The response of grafted embryonic tissue is similar to that of neoplastic tissues and different from that of placental tissue, which will not grow even in mice of the homologous pure line; but in general the defence reactions to implanted tissue are governed by genetically determined antigenic differences between the implanted tissue and the host. Experiments on resistance to the Jensen rat sarcoma carried out at the Middlesex Hospital by Prof. S. Russ and Dr. G. M. Scott have shown that the immunity induced by treatment of tumour-bearing rats with $\mathrm{X}$-rays is inherited by the offspring. This appears to be an example of inheritance of an induced or acquired character.

By far the largest individual account in this year's report is that from Dr. P. Stocks, of the General Register Office, Somerset House, on the distribution of cancer of various organs in England and Wales. This account, which is the third and concluding instalment, deals with the cancer mortality of the female population and is more of the nature of a scientific paper than an interim report of work in progress. The standardized mortality ratios for separate towns and counties have now been computed and the results are given in a series of tables and maps. As would be expected from analogous investigations, the mortality from cancer of all sites varies much less than the mortality due to cancer of particular sites. Thus the cancer mortality of part of Wales (Anglesey, Carnarvon, Denbigh, Flint and Merioneth) and of Lincolnshire is higher than that of the rest of the country; but the excess mortality is only of the order of 15 per cent. The high cancer mortality of North Wales is entirely accounted for by an excess of stomach cancer, affecting both sexes. Dr. Stocks suggests that this excess, which is about one and a half times the expected mortality, is due to dietary factors. It is hinted that the bulk of the North Wales population does not consume sufficient vegetables and fruit, and uses the frying-pan too frequently. If this mortality from gastric cancer could be reduced, people escaping might contract cancer at some other site, which might be more amenable to treatment, and some of them would probably avoid cancer altogether.

In the results are several examples of low mortality from cancer of one particular site being compensated for by excessive incidence at somo other site. Thus there is a high incidence of uterine cancer in the north-east of England, and in this area cancer of the breast is correspondingly low. Analogous differences are found in the social classes. The incidence of cancer of the uterus in married women decreases steadily with rise in 
social class, while cancer of the breast is more frequent in the upper classes. This may be connected with birth-rate, as fertility varies inversely with inecme Also it is known that countries with high birth-rates tend to have higher relative proportions of uterine and lower proportions of breast cancer ; cancer of the mouth, pharynx, œsophagus, skin and vagina, like uterine cancer, are more common in the lower classes, while cancer of the ovary and thyroid, like breast cancer, are more common in the upper classes.

For almost all sites of cancer that are examined (except cancer of the ovaries) the urban incidence is higher than that in rural districts. The figures for cancer of the lung in different districts are difficult to explain; the incidence being very high in Manchester, Leeds, Sheffield and Birmingham, but low in other towns in Lancashire, Durham and Stoke-on-Trent.

The results of Dr. Stocks's investigations have been dealt with at some length partly because it is conceivable that men of science who are not specially working at the cancer problem might see some explanation of the regional distribution of cancer mortality. Such explanations might give rise to fruitful research on the cause of the disease at a particular site.

Until the outbreak of war, British cancer research had been increasing in its extent and effectiveness, as is clearly shown by the annual reports of the British Empire Cancer Campaign. Let us hope that the work will continue and that the present position will be consolidated and extended in the immediate future.

\section{MACROMOLECULES}

SOME developments in the chemistry and physics of macromolecules were discussed at a recent meeting of the Plastics Group of the Society of Chemical Industry and the Faraday Society. Many fundamental problems were raised in the papers contributed by Dr. R. Bhattacharya, E. G. Couzens and H. W. Melville, and in the subsequent discussion; only a few can be referred to in the present article.

In the synthesis of macromolecules from vinyl derivatives of the general type $\mathrm{CH}_{2}=\mathrm{CXY}$, much controversy has arisen about the nature of the active polymer molecules which react with the moncmer. Two extreme types may be recognized. The monomer may be converted by absorption of energy to a diradical. To each end of this molecule monomeric molecules may add on, giving rise to a bigger diradical. This may be called the free radical mechanism. On the other hand, the moncmer may merely be excited to an extent such that the addition of monomer is facilitated. In this mechanism there is always a terminal double bond in the macromolecule which is the centre of reactivity. It would now appear that it is possible to polymerize one and the same molecule by either mechanism, depending on conditions.

The difficulty really arises in discovering in any one instance which particular mechanism comes into operation. In gas phase polymerization it is often possible to settle the matter in the following way. Hydrogen atoms or methyl radicals add on readily to vinyl derivatives, such as vinyl acetate, producing a single free radical with which monomer easily reacts to form a linear polymer until two such radicals combine. By altering various parameters, it is then easy to characterize this free radical growth and compare it with the polymerization under observation. If the kinetics are identical, it must be supposed that the latter reaction is of the free radical type. In the photo-polymerization of gaseous vinyl acetate, the kinetics are so different from those of the free radical reaction that it would seem the ordinary polymerization occurs by some double-bond mechanism. The difficulty yet to be solved is how the terminal double bond of the active molecule retains its activity. It is well known that a large number of these molecules, including vinyl acetate, also polymerize in the liquid phase, but so far no similar attempt has been made to discover by what mechanism the molecules grow, though it is of interest to mention that a free radical polymerization has been induced in styrene by the addition of tetraphenylsuccinodinitrile, which dissociates to free radicals in solution.

From the photochemical point of view, vinyl ketones are of especial interest. Both methyl vinyl ketone $\left(\mathrm{CH}_{2}=\mathrm{CH} . \mathrm{CO} . \mathrm{CH}_{3}\right)$ and methyl isopropenyl ketone $\left(\mathrm{CH}_{2}=\mathrm{C}\left(\mathrm{CH}_{3}\right) \cdot \mathrm{CO} \cdot \mathrm{CH}_{3}\right)$ polymerize on illumination with light at $3000 \mathrm{~A}$., though the former molecule does so much more readily than the latter. Once more this is in accord with the general observation that $\alpha$-methyl substituted vinyl derivatives are more difficult to polymerize than the parent molecule. Methyl vinyl ketone exhibits rather peculiar behaviour. Like most gaseous ketones, it is decomposed by light, and therefore in considering the mechanism of poly. merization, it is not impossible that, besides the two mechanisms mentioned above, another must 